Meta

Journal des traducteurs

Translators' Journal

\title{
La théorie de la traduction en Espagne
}

\section{Fernando Navarro Domínguez}

Volume 55, numéro 1, mars 2010

Le parcours du sens : d'une langue à l'autre — Mélanges offerts à André Clas

The Way of Meaning: From a Language to Another - Collection of Articles Offered to André Clas

URI : https://id.erudit.org/iderudit/039604ar

DOI : https://doi.org/10.7202/039604ar

Aller au sommaire du numéro

\section{Éditeur(s)}

Les Presses de l'Université de Montréal

ISSN

0026-0452 (imprimé)

1492-1421 (numérique)

Découvrir la revue

Citer cet article

Navarro Domínguez, F. (2010). La théorie de la traduction en Espagne. Meta, 55(1), 88-104. https://doi.org/10.7202/039604ar

\section{Résumé de l'article}

Le présent article fait état d'un aperçu de la réflexion, en matière de théorie de la traduction, d'un important groupe de professeurs d'universités espagnoles qui oeuvrent en traduction ou en traductologie. Dans les années 1990, une série d'ouvrages pionniers voient le jour et offrent une riche diversité de concepts sur la théorie de la traduction et sur son application didactique. Si, pour Lvóvskaya, il convient de mettre l'accent sur l'équivalence dans la communication bilingue, pour Elena, il s'agit plutôt d'appliquer à l'espagnol les théories allemandes du fonctionnalisme et du skopos. Tricás, quant à elle, développe l'application des théories de l'argumentation linguistique en traduction sur le plan textuel, tandis que Hurtado se centre sur un développement exhaustif de chacun des piliers de la traduction. Rabadán et Merino, de leur côté, centrent leurs travaux sur les Études descriptives de traduction (EDT). Par ailleurs, d'autres chercheurs suivent des orientations diverses : Vidal (déconstruction, manipulation, pouvoir), Carbonell (postcolonialisme), Corpas (corpus traductologiques), Muñoz (dimension cognitive), Ortega (herméneutique) et Mayoral (la traduction comme technologie).
Ce document est protégé par la loi sur le droit d'auteur. L’utilisation des services d'Érudit (y compris la reproduction) est assujettie à sa politique d'utilisation que vous pouvez consulter en ligne.

https://apropos.erudit.org/fr/usagers/politique-dutilisation/ 


\title{
La théorie de la traduction en Espagne
}

\author{
FERNANDO NAVARRO DOMÍNGUEZ \\ Université d'Alicante, Alicante, Espagne \\ tra.fnavarro@ua.es
}

\section{RÉSUMÉ}

Le présent article fait état d'un aperçu de la réflexion, en matière de théorie de la traduction, d'un important groupe de professeurs d'universités espagnoles qui œuvrent en traduction ou en traductologie. Dans les années 1990, une série d'ouvrages pionniers voient le jour et offrent une riche diversité de concepts sur la théorie de la traduction et sur son application didactique. Si, pour Lvóvskaya, il convient de mettre l'accent sur l'équivalence dans la communication bilingue, pour Elena, il s'agit plutôt d'appliquer à l'espagnol les théories allemandes du fonctionnalisme et du skopos. Tricás, quant à elle, développe l'application des théories de l'argumentation linguistique en traduction sur le plan textuel, tandis que Hurtado se centre sur un développement exhaustif de chacun des piliers de la traduction. Rabadán et Merino, de leur côté, centrent leurs travaux sur les Études descriptives de traduction (EDT). Par ailleurs, d'autres chercheurs suivent des orientations diverses: Vidal (déconstruction, manipulation, pouvoir), Carbonell (postcolonialisme), Corpas (corpus traductologiques), Muñoz (dimension cognitive), Ortega (herméneutique) et Mayoral (la traduction comme technologie).

\section{ABSTRACT}

The present paper provides an overview of the reflections about translation theory carried out by a large group of Spanish university scholars working in the fields of Translation and Translation Studies. A number of pioneering works in these fields that offer various and rich concepts about translation theory and its didactic applications were published in the 1990s. While Lvóvskaya emphasizes on equivalence in bilingual communication, Elena is more inclined to apply functionalist and skopos German theories to Spanish language and translation. On the other hand, Tricás explores the application of language argumentation theories to translation on a textual level, whereas Hurtado pursues an exhaustive study of every pillar of translation, and Rabadán and Merino focus on the Descriptive Translation Studies (DTS). This paper also mentions the work of Vidal (deconstruction, manipulation, power), Carbonell (postcolonialism), Corpas (translation corpus), Muñoz (cognitive dimension), Ortega (hermeneutics) and Mayoral (translation as technology).

\section{MOTS-CLÉS/KEYWORDS}

fonctionnalisme, argumentation, textualité, cognitivisme, descriptivisme functionalism, argumentation, textuality, cognitivism, descriptivism

\section{Introduction}

À l'occasion du premier congrès de linguistique appliquée tenu à l'Université de Murcie, Santoyo (1983: 265) amorçait avec sa translémique la polémique quant au nom à donner en Espagne aux unités de traduction et à la discipline. De nos jours, les deux noms les plus utilisés pour nommer cette discipline sont translation studies et traductologie. Le premier est employé dans le monde anglo-saxon, tandis que le second jouit d'une grande acceptation dans le monde francophone. Les principaux 
auteurs espagnols qui adoptent plutôt une traduction littérale du premier (estudios de traducción) sont Santoyo, Rabadán, Merino, Nistal, Calzada et Mayoral, tandis que ceux qui favorisent le second (traductología) sont, entre autres, Hurtado, Tricás ou Peña. Si la désignation de la discipline engendre des divergences, les chemins parcourus par les différentes théories à propos de la nature de la traduction ne sont pas en reste.

L'objectif du présent article est d'offrir une synthèse de l'effort réalisé par un vaste groupe de chercheurs espagnols en matière de théorie de la traduction et de ses possibles applications en didactique. Cet effort se matérialise par des propositions (et des réécritures) qui complètent, corrigent ou réécrivent, sous un nouveau jour, la théorisation engendrée par les auteurs européens et nord-américains. Certaines approches théoriques ouvrent de nouvelles voies et suscitent la controverse chez les chercheurs.

Les ouvrages reflétant les travaux des chercheurs dans le domaine de la théorie ne manquent pas en Espagne (entre autres, Textos clásicos de teoría de la traducción [Vega Cernuda 1994/2004], Traducción y Traductología. Introducción a la Traductología [Hurtado 2001], Teoría de la traducción: convergencias y divergencias [Sánchez Trigo 2002], La selva de la traducción [Moya 2004], Panorama actual de la investigación en traducción e interpretación [Ortega 2004] et El espejo traductológico [Calzada 2007]). Toutefois, aucun d'entre eux n'analyse les travaux théoriques réalisés exclusivement en espagnol, car ils se penchent sur les grands noms d'origine étrangère plutôt que sur les auteurs espagnols. Telle est la lacune que nous nous proposons de combler ici.

Dès les balbutiements de la réflexion théorique en traduction, dans la seconde moitié du $\mathrm{xx}^{\mathrm{e}}$ siècle, les chercheurs espagnols se sont d'abord efforcés de proposer une théorisation et des contenus didactiques conjuguant théories et principes didactiques, puis de donner à leurs travaux une certaine visibilité à partir des années 1990, grâce à des ouvrages tels que Manual de bibliografía española de traducción: Diez años de historia 1985 - 1995 (Navarro 1996) ou Bibliografía de traducción en español, catalán, gallego y vasco (Santoyo 1996). Les nombreuses monographies et actes de congrès publiés en Espagne, les colloques, séminaires et conférences tenus au pays, ainsi que l'existence d'une vingtaine de revues de traduction témoignent de l'énorme effort des chercheurs et professeurs espagnols visant à la constitution d'une nouvelle discipline universitaire dotée de cadres théoriques, descriptifs et appliqués.

Nous examinons ici un choix nécessairement restreint d'auteurs espagnols qui donnent de la théorie de la traduction une définition propre ou qui en proposent une réécriture.

Réécrire le concept de théorie de la traduction à partir de la contribution hispanique suppose l'ouverture d'un éventail de voies qui ne se croisent pas toujours, puisque le centre d'intérêt peut être constitué par les langues, la nature communicative des textes, la finalité de la communication, le lien entre traduction et culture d'arrivée, la typologie des corpus, etc. L'analyse sera articulée en trois parties: les travaux pionniers des années 1980, les publications des années 1990, qui alimentent l'essentiel de notre réflexion et, finalement, les contributions de ce début du $\mathrm{XXI}^{\mathrm{e}}$ siècle. 


\section{Les premiers travaux de théorisation de la discipline. Deux grands pionniers: Valentín García Yebra et Julio César Santoyo}

Plusieurs événements majeurs marquent le début de la réflexion hispanique sur la théorie de la traduction dans les années 1980. En premier lieu, la création de la Fundación Alfonso X el Sabío, à Madrid, avec le soutien du ministère de la Culture espagnol et de professeurs prestigieux, dont Emilio Lorenzo, Jesús Cantera, Valentín García Yebra, Francisco Hernández... Dans la préface de Problemas de traducción, Mesa Redonda, tenue à Madrid en 1983, Emilio Lorenzo reprend certains problèmes théoriques de la traduction et explique:

[...] nació con la vocación de ser cauce de iniciativas y lugar de encuentro para quienes hayan llegado al convencimiento de que velar por la calidad de la traducción y por la mejor formación de los traductores es tanto como hacerlo por la calidad y corrección del idioma y por la riqueza de los intercambios entre los pueblos (Lorenzo 1987: 5).

L'auteur le plus important de cette période est, sans aucun doute, Valentín García Yebra, devenu académicien de la Real Academia Española en 1987 pour sa contribution à la traduction. Il a laissé des ouvrages fondamentaux tels que Teoría y práctica de la traducción (1982), En torno a la traducción (1983/1989) et Traducción: Historia y teoría (1994), sans oublier son important Diccionario de galicismos prosódicos y morfológicos (1999). Dámaso Alonso, poète, académicien et directeur de la Real Academia Española, préfaçant l'œuvre pionnière de García Yebra, Teoría y práctica de la traducción, rappelle que l'Espagne, pays qui publia, en 1980, 7800 traductions (27 629 livres publiés au cours de cette année-là) principalement de l'anglais, du français, de l'allemand et de l'italien, avait besoin d'un ouvrage tel que celui-là qui puisse servir de guide. Pourtant, au moment de sa publication, les intentions de García Yebra étaient plutôt modestes:

[...] ofrecer un conjunto de ideas, al mismo tiempo, de indicaciones prácticas, que les allanen el camino a los traductores y les permitan conseguir más seguridad, mayor soltura y mejor calidad en su trabajo (García Yebra 1982: 13).

En ce qui concerne les concepts clés de traduction, García Yebra, d'une part, cite des auteurs tels que Taber et Nida, Vinay et Darbelnet, Malblanc, Mounin, Wandruszka et, d'autre part, centre sa théorie sur la traduction écrite littéraire. Dans le cadre de notre brève évocation, citons ses affirmations selon lesquelles «la teoría y la práctica de la traducción son inseparables de la enseñanza de la disciplina» et «el estudio de la traducción es una rama de la lingüística aplicada» (García Yebra 1982: 18). Il reprend la définition de traduction de Taber et de Nida (1971: 11; cité dans García Yebra 1982: 14), mais surtout celle de Dubois, tirée de son Dictionnaire de linguistique: "Traduire c'est énoncer dans une autre langue (ou langue cible) ce qui a été énoncé dans une langue source, en conservant les équivalences sémantiques et stylistiques» (Dubois 1973: 490; cité dans García Yebra 1982: 13). Il la reprendra d'ailleurs dans Traducción: historia y teoría (García Yebra 1994: 305-306), pour faire référence à la traduction en tant que processus et énoncer sa propre définition: traduction écrite et non orale, traduction interlinguistique, «a la que llamaré traducción sin más determinaciones». Finalement, dans un ouvrage plus récent, intitulé Experiencias de un traductor (García Yebra 2006), il affirme que ses travaux, qui s'intéressent surtout au processus, relèvent de la théorie de la traduction, tandis que 
l'étude de la traduction en tant que produit fait plutôt partie de la critique de traductions.

Réécrire la théorie de la traduction à partir d'une vision historique et critique est une tâche difficile qui requiert un travail approfondi de documentation. Julio César Santoyo, professeur à l'université de León, l’a accomplie pour nous faire découvrir l'histoire grâce à de nombreuses publications. En 1987, Santoyo affirmait:

La traducción entra ya en 1965 (por lo que a España se refiere) en las bibliografías propiamente dichas, emancipándose así del carácter secundario, incidental e independiente que hasta entonces había tenido. [...] Nadie ha abordado aún la tarea de recopilar, comentar y ponderar lo que en España e Hispanoamérica se ha escrito sobre la traducción desde 1963 (Santoyo 1987: 14).

Dix ans plus tard, il écrit encore:

Los estudios de traducción, sobre todo los de condición teórica, han experimentado en los tres últimos decenios un desarrollo cualitativa y cuantitativamente espectacular, que no conoce parangón en otras ramas del saber lingüístico (Santoyo 1997: 99).

Santoyo nous avait déjà surpris avec des travaux comme La cultura traducida (1983) et El delito de traducir (1985/1996). Parcourir les travaux de Santoyo qui ont contribué à l'étude diachronique de la théorie de la traduction nous mène surtout à sa Teoría y crítica de la traducción (1987) qui présente une compilation de presque cent traducteurs et théoriciens du monde hispanique. La richesse des références fait de cet ouvrage un incontournable des études de traduction dans le monde hispanique. Dans son article intitulé Del scriptorium a la Academia: acerca de cómo, dónde, cuándo y por qué la reflexión teórica se ha ido incorporando al quehacer traductor, Santoyo souligne l'évolution de la discipline:

A finales de los años 50 comienza, pues, el cuarto período de esta historia, en el que ahora estamos, caracterizado en términos muy sencillos por un cambio acelerado que ha transformado la "literatura" de la traducción en "ciencia" de la traducción (Santoyo 1997: 104).

Par ailleurs, à la question de savoir pourquoi les études théoriques doivent être présentées dans le programme, essentiellement orienté vers la pratique, d'un centre universitaire de traduction et interprétation, il répond:

Porque el quehacer del traductor ya no puede prescindir de una base teórica que de hecho sustenta y condiciona toda la práctica. Porque como señala M. Ballard (1993: 11) en su obra La traduction à l'université, lo propio de un profesor universitario es la investigación epistemológica (Santoyo 1997: 104).

Comment Santoyo envisage-t-il le concept de traduction? Pour lui,

[1]a traducción es el resultado textual de varias formas complejas de transcodificación lingüística, mediante las cuales, y conservando todo lo posible los aspectos formales y textuales, se reconvierte un TL1 (texto en la lengua origen) en otro TL2 (texto en la lengua meta) dialécticamente derivado de él (aunque sometido en el proceso a distintos condicionamientos culturales, lingüísticos y/o mediáticos) y funcionalmente equivalente en el PS2 (polisistema meta) al TL1 en el PS1 (polisistema origen) (Santoyo, communication personnelle ${ }^{1}$ ). 


\section{Les années 1990 : entre théorisation et développement de la didactique}

\subsection{Les théories communicatives: l'équivalence communicative de Lvóvskaya}

Zinaida Lvóvskaya a mené un travail important présenté dans Problemas actuales de la traducción (1997). Cette professeure russe, qui a enseigné à la Universidad de Las Palmas de Gran Canarias durant de nombreuses années, exprime ainsi sa perception du stade auquel est rendue la science de la traduction:

Todos los autores coinciden en que el objeto de la Ciencia de la Traducción es la comunicación bilingüe que, aparte de tener las características generales de cualquier proceso de comunicación verbal, posee sus características específicas que garantizan estatus científico a los estudios traductológicos. Es precisamente por ello por lo que muchos autores, al definir el proceso de la traducción, destacan, unas veces, las características generales de toda comunicación verbal, y otras, las características específicas de la comunicación bilingüe equivalente [...]. Estas definiciones parten de las características universales de la comunicación verbal y por tanto son aplicables tanto a la comunicación monolingüe, como a la bilingüe, tanto a la comunicación bilingüe equivalente, como a la heterovalente (Lvóvskaya 1997: 95-96).

L'auteure signale un peu plus loin que, bien que les études traductologiques soient menées, à ce moment-là - nous sommes en 1997 -, sous un angle communicatif, on ne peut affirmer que leur objet soit totalement clair, ni leur cadre conceptuel suffisamment équilibré.

A juzgar por los trabajos de los partidarios de la skopos théorie y de la Escuela de Tel Aviv, se pone en entredicho [...] que es la equivalencia comunicativa de dos textos, lo que conduce a consecuencias paradójicas para la Ciencia de la Traducción, haciéndola prácticamente imposible, puesto que una teoría no puede describir y explicar científicamente dos tipos de actividades diferentes en sus objetivos y por lo tanto en sus estructuras, recursos, requisitos que debe reunir el producto final, competencias del sujeto de la actividad y factores que la determinan (por lo menos si se parte de la teoría de la acción). Creemos que para ello se necesitan dos teorías (si no más) cada una de las cuales tenga su propio problema y sus propias sugerencias para solucionarlo (Lvóvskaya 1997: 97).

Cette situation ambiguë, selon l'auteure, vient de ce que les partisans de la théorie communicative mettent au premier plan des caractéristiques du processus de traduction telles que le caractère cognitivo-communicatif et intersubjectif du processus traductionnel ou le rôle dominant de la situation communicative dans l'interprétation et la production du texte. Mais ces caractéristiques ne sont d'aucune utilité pour définir une théorie de la traduction, car elles ne sont pas exclusives de cette discipline ni de la théorie générale de la communication. Pour l'auteure, «la única característica exclusiva de la traducción es la relación de equivalencia comunicativa que deben guardar el TO y el TM» (Lvóvskaya 1997: 98).

Bien qu'elle reconnaisse que Rabadán (1991: 232) a raison d'affirmer que «todo intento de reducir los fenómenos de traducción a categorías fijas establecidas según criterios estáticos conduce únicamente a la distorsión de la realidad», Lvóvskaya affirme que l'on peut formuler ainsi des normes communicatives:

Normas comunicativas (cognitivo-culturales) que no reducen los fenómenos de traducción a categorías fijas, ni establecen criterios estáticos, sino que delimitan de cierta manera (comunicativamente) las opciones del traductor, sirviendo de filtros que restringen su arbitrariedad y establecen límites (Lvóvskaya 1997: 99). 
Lvóvskaya (1997: 101) défend la primauté de la théorie sur d'autres aspects de la traduction: "por inobservable que sea el objeto de una ciencia, el lugar jerárquico más importante corresponde a la teoría». Bien qu'elle reconnaisse que toute science a trois fonctions (expliquer, décrire et prédire), elle considère que les différences théoriques particulières de la traduction sont peu développées et que la réflexion théorique doit s'appuyer non seulement sur des études descriptives et appliquées pour confirmer ou réfuter les hypothèses théoriques, mais également sur la réflexion ou la logique scientifique, comme le montrent d'autres sciences.

Afin d'expliquer scientifiquement et de formuler les normes communicatives du comportement du sujet dans le cadre d'une activité donnée, les données empiriques ne sont pas suffisantes, en particulier lorsqu'il s'agit d'une activité intersubjective:

Se necesita una teoría coherente, aunque sea una hipótesis científica que, debe encontrar, desde luego, su confirmación, en estudios empíricos y aplicados. Precisamente por ello no se puede calificar la Teoría General de la Traducción de disciplina empírica (Lvóvskaya 1997: 105).

Lvóvskaya indique que l'approche de la théorie générale de la traduction analysée dans ses travaux s'appuie sur deux prémisses: d'une part, la différence entre contenu et sens d'un texte et, d'autre part, le concept d'activité défini par la théorie de l'action. La première proposition mène au concept d'équivalence communicative de deux textes produits dans différentes cultures. Avec la seconde, elle conclut que deux activités ayant des objectifs différents ne peuvent être expliquées scientifiquement par une même théorie. En matière d'activité de communication bilingue interculturelle, le concept de base est l'équivalence communicative, trait essentiel de la délimitation du concept de traduction.

\subsection{Le fonctionnalisme et la théorie du skopos}

L'approche théorique du fonctionnalisme et du skopos est essentiellement représentée par Reiss, Vermeer et Nord. Dans ce cadre, il convient de souligner l'importante contribution de Pilar Elena, de l'université de Salamanque, qui a su articuler une intéressante proposition méthodologique - de nos jours largement acceptée - et l'appliquer à la traduction allemand-espagnol. Elena propose une étude globale du texte qui tient compte de sa macrostructure et de sa microstructure. Dans la macrostructure, des aspects tels que l'émetteur, l'intention, le récepteur, le temps, le lieu, le moyen ou encore la fonction textuelle, font l'objet d'une attention toute particulière. L'étude de la microstructure, quant à elle, se centre sur les aspects sémantiques (thématique, contenu) et formels (aspects verbaux et non verbaux). Elena construit un modèle de typologie textuelle qui permet de rendre, dans chaque situation, la traduction la plus adéquate possible. Elle expose ces principes dans trois ouvrages où elle développe sa proposition didactique: Aspectos teóricos y prácticos de la traducción alemán-español (Elena 1990), Curso práctico de traducción general alemán-español (Elena 1994) et El traductor y el texto (Elena 2001). Dans le premier, l'auteure rappelle:

La lista de definiciones parecidas - las que ha explicado previamente - podría prolongarse llenando varias páginas con ellas, sin embargo, no ayudarían a llegar al fondo de la problemática relacionada con el proceso de la traducción. Por este motivo he preferido centrarme en aquéllas que [...] se centran en los aspectos lingüísticos de la tra- 
ducción, y las que tienen en cuenta también los aspectos pragmáticos de la misma (Elena 1990: 15).

Elle conclut, en évoquant un texte de Nord (1987: 5; cité dans Elena 1990: 61):

la teoría de la traducción debería partir de esta base [los elementos integrantes de la competencia traductora] para el desarrollo de unas técnicas o procedimientos encaminados a la solución de los problemas de toda índole que surgen al traducir. De esta forma el núcleo de la cuestión se ampliaría al considerar tanto el texto en su globalidad como las fuentes de información acerca del texto (Elena 1990: 61).

Dans son deuxième ouvrage paru en 1994, elle approfondit les aspects pratiques de la traduction allemand-espagnol en vue d'un cours de traduction générale, base de l'apprentissage de la traduction, tout comme le signalait Lvóvskaya. Enfin, dans son troisième, paru en 2001, Elena énumère une série d'objectifs d'apprentissage fondés sur le concept de typologie textuelle et définit le type d'adéquation le plus opportun. Les trois types de textes sont les suivants: informatifs (informations de presse), appellatifs (publicitaires) et expressifs (littéraires). Son approche pédagogique prévoit des tâches individuelles et en groupe, ces dernières constituant une des nouveautés et un des attraits de la proposition inspirée de la théorie de didactique générale qui cherche à développer des ressources motivantes pour les étudiants.

Enfin, qu'est-ce que traduire selon Elena? Elle y répond dans un article paru en 2006:

Traducir es un proceso cognitivo complejo cuyos componentes principales abarcan esa doble vertiente: la recepción textual y la (re)producción textual. Ambas actividades se caracterizan por un trabajo selectivo y electivo que está muy condicionado por la competencia traductora global (Elena et De Kock 2006: 144).

\subsection{Argumentation linguistique, analyse textuelle et analyse du discours}

Mercedes Tricás, professeure à la Universitat Pompeu Fabra à Barcelone, présente un autre modèle théorique et pratique de la traduction, appliqué à la combinaison linguistique français-espagnol. Ce modèle s'inspire de l'analyse du discours, de la linguistique textuelle et de la pragmatique. L'auteure, au début de son ouvrage intitulé Manual de traducción francés-castellano, définit le concept de traduction de la façon suivante:

Resumiendo pues, la traducción consiste estrictamente en un acto de comunicación que pretende reproducir el sentido de un mensaje, mediante la creación, en otra lengua, de un mensaje equivalente, con una función comunicativa similar, expresado en la forma más adecuada posible, para que pueda ser entendido por un nuevo lector en una nueva situación (Tricás 1995/2003: 203).

Elle rappelle que les théories de la traduction peuvent être divisées en deux grands groupes: celles qui mettent l'accent sur l'aspect purement linguistique de l'opération de transfert, et celles qui se centrent sur l'aspect communicatif. Ce second groupe comprend notamment, selon elle, la théorie connue sous le nom de théorie du sens, développée à l'ÉSIT (École supérieure d'interprétation et de traduction), à Paris, accompagnée des principes mis de l'avant par Delisle (1980) dans L'analyse du discours comme méthode de traduction. Un des aspects les plus intéressants de l'ouvrage de Tricás nous paraît cependant être l'application de ces théories à des 
problèmes pratiques de la traduction français-espagnol dans les chapitres trois, quatre et cinq.

Les principes proposés par Tricás correspondent à trois étapes du processus: l'interprétation, la restitution et la révision. En ce qui concerne l'interprétation, elle établit un parcours réflexif allant du contexte communicatif au macrotexte, en passant par le microtexte (cohésion lexicale); pour la restitution, elle considère que le traducteur doit se pencher sur certaines techniques comme les transpositions ou les modulations, les adéquations culturelles, les normes linguistiques et les restitutions graphémiques; pour la révision du texte traduit, elle propose une longue liste d'instructions qu'elle articule autour de l'adéquation de la charge pragmatique fonctionnelle relative à l'unité textuelle globale.

Tricás est aussi l'auteure de nombreux articles portant sur la traduction, la linguistique textuelle et la pragmatique. Après Lingüística textual y traducción (1987a) (primé par la Fundación Alfonso X el Sabio), elle a notamment publié La traducción de los conectores del discurso (con referencia a la traducción del francés al castellano (Tricás 1987b), L'argumentation concessive française et espagnole: le transfert en espagnol de quelques connecteurs (Tricás 1990), Polifonía discursiva y traducción (Tricás 1991), La traducción al español de los valores interactivos y argumentativos del conector «alors» (Tricás 1995) et Construir (equivalencias): del texto a la representación intercultural (Tricás 2008).

\section{Cinq pistes pour la recherche traductologique au $\mathrm{XXI}^{\mathrm{e}}$ siècle}

Les cinq axes de recherche présentés ci-après ne naissent pas au $\mathrm{xxI}^{\mathrm{e}}$ siècle mais suscitent actuellement l'intérêt de nombreux chercheurs:

1) l'intégration des théories dans une approche globale;

2) les études descriptives de traduction;

3) les théories fondées sur la manipulation, la déconstruction, l'éthique et le postcolonialisme;

4) les nouvelles technologies et les corpus;

5) l'émergence du cognitivisme.

\subsection{Une approche globale de la traduction}

Amparo Hurtado, professeure à la Universitat autónoma de Barcelona (UAB), propose une analyse intégratrice et globale de la traduction. Selon elle, en effet, toute définition de la traduction doit nécessairement prendre en compte: le texte, l'acte de communication et l'activité cognitive du sujet. Sa définition de la traduction est donc la suivante:

Un proceso interpretativo y comunicativo consistente en la reformulación de un texto con los medios de otra lengua que se desarrolla en un contexto social y con una finalidad determinada (Hurtado 2001: 41).

L'auteure présente l'analyse de chacune des trois composantes de la traduction de la façon suivante:

a) La traduction comme activité cognitive, soit l'étude du processus de traduction et de la compétence du traducteur. Elle étudie les multiples modèles d'analyse et 
souligne que l'étude du processus est ardue, car on doit prendre en compte six prémisses essentielles:

La existencia de procesos básicos en el desarrollo del proceso traductor. El carácter interactivo y no lineal del proceso traductor. La existencia de procesos controlados y no controlados. La consideración del proceso traductor como un proceso de reconocimiento y resolución de problemas. Y la existencia de características específicas del proceso traductor en cada modalidad y tipo de traducción según el método elegido (Hurtado 2001: 367-371).

En ce qui concerne la compétence traductionnelle, après avoir étudié différentes propositions, Amparo Hurtado présente le modèle PACTE élaboré à la UAB (2001: 385).

b) La traduction en tant qu'opération textuelle est étudiée à partir de différents modèles:

La traducción como operación textual es estudiada desde la descripción de diferentes modelos de análisis y desde las diferentes tipologías textuales [...]. La textología comparada y las diferentes aplicaciones al análisis textual al estudio de la traducción: superestructura, macroestructura y microestructura; criterios de textualidad de Neubert, textura y traducción de Hatim y Mason y Baker [...] (2001: 409-506).

c) La traduction en tant qu'acte de communication peut être expliquée à partir de différents modèles:

La traducción como acto comunicativo se explica con el estudio de ocho modelos y termina con los factores de análisis que son los siguientes: Las diferencias de uso, las diferencias de usuario, la historicidad, el contexto sociocultural, la ideología, los estudios poscoloniales y la traducción y feminismo (Hurtado 2001: 506-626).

Toutefois, le travail d'Hurtado n'est pas limité à l'ouvrage mentionné. Ses inquiétudes relatives à l'apprentissage de la traduction et à la didactique utilisée dans les centres universitaires l'ont menée à développer une recherche en équipe sur ce sujet. Ces travaux visent à analyser les parcours possibles en didactique de la traduction, à l'aide de manuels aux objectifs d'apprentissage clairs. Ses travaux dans ce domaine (Hurtado 1996; 1999) constituent actuellement un modèle pour tous ceux qui désirent approfondir leurs connaissances en didactique de la traduction et sur les objectifs d’apprentissage reliés à cette discipline.

\subsection{Les études descriptives de traduction}

Les travaux d'Itamar Even-Zohar et de Gideon Toury, professeurs à Tel Aviv, sont connus en Espagne depuis longtemps. Ces auteurs sont intervenus à plusieurs reprises dans différents forums universitaires afin d'expliquer leur conception des études descriptives de traduction (EDT). Parmi les principaux ouvrages, il convient de citer: Toury (1997) et Even-Zohar (1999) ainsi que la traduction en espagnol de l'ouvrage de Toury (1995) par Rabadán et Merino (2004), Descriptive Translation Studies and Beyond.

Les mots clés de la théorisation de Zohar et Toury sont normes et idiosyncrasies. Les normes constituent, dans les polysystèmes, des comportements habituels qui ont une influence socioculturelle sur ceux qui les emploient. Les traductologues descriptifs s'attellent à identifier les normes et les comportements en traduction. 
Rosa Rabadán et Raquel Merino, professeures à la Universidad de León et à la Universidad del País Vasco, présentent ainsi, dans la préface de leur traduction de l'ouvrage de Toury, le concept d'études descriptives en traduction (EDT):

Los EDT son la pieza central y la base de los Estudios de Traducción (Rabadán et Merino 2004: 18).

Los EDT son el puente entre la teoría y la práctica, el necesario punto de partida hacia los estudios aplicados (o extensiones aplicadas), así como un viaducto de ida y vuelta entre la práctica real existente y la que los traductores producirán en el futuro [...] el nuevo marco de los Estudios de Traducción supone pasar de afirmaciones gratuitas a establecer un protocolo de trabajo basado en el esquema habitual del método científico (hipótesis - observación empírica - verificación (Rabadán et Merino 2004: 19).

Los Estudios Descriptivos de Traducción (EDT) son sin duda una potente metodología que ha permitido secuenciar los distintos pasos de la investigación básica en Estudios de Traducción (Rabadán et Merino 2004: 21).

D'autres travaux de Rabadán (2000) et Merino (2001) sur la censure et la traduction dans l'Espagne franquiste sont aussi très importants.

\subsection{Théories déconstructivistes, manipulation, pouvoir et postcolonialisme}

África Vidal, professeure à la Universidad de Salamanca, étudie les courants systémiques et descriptifs. Elle analyse les propositions idéologiques de Basnett et de Lefevere, présente les théories déconstructivistes et féministes et analyse l'éthique du traducteur en s'inspirant de Derrida et de Foucault (Vidal 1995; 1998; 2005; 2008). L'auteure propose un cadre d'analyse traductologique hybride, centré sur des théories antérieures et sur la théorie philosophique de Foucault. La proposition comporte trois composantes: une archéologie du savoir (la description rigoureuse des phénomènes de traduction); une généalogie du pouvoir (le contenu idéologique qui structure la traduction ou la rend impossible); et une éthique transversale qui octroie aux traducteurs le rôle de moteur de changement. Selon Vidal:

El llamado giro cultural de la traducción nos hizo conscientes de la importancia del concepto socio-político y de cuestiones ideológicas y de manipulación que están siempre presentes en el acto de traducir (Vidal 2008: 79).

Y precisamente esa visión de la traducción que incorpora conceptos como el de la microfísica de Poder de Michel Foucault, el de ideología en el sentido de Lefevere o el del traductor como lector de Hans-Georg Gadamer, por citar solo algunos, la que ha dado pie a la construcción de teorías tan interesantes como las teorías postcoloniales de la traducción [...] o las teorías feministas [...] que han hecho correr ríos de tinta, porque incorporan a la investigación en traducción "broad questions about ideology, ethics and culture" (Vidal 2008: 82).

L'auteure ne donne pas de recettes définitives; elle fait plutôt état d'une argumentation théorique solide qui prévoit l'adaptation aux circonstances du moment et qui fait écho à des auteurs comme Tymoczko, Venuti ou Lefevere qui ont inspiré de nombreux chercheurs. Vidal procède ainsi à une réécriture prometteuse de la traduction. Elle conclut de la façon suivante un récent article: 
La traducción en el siglo XXI es una condición permanente del ser humano que se refleja en una práctica discursiva que releva los múltiples signos de la polivalencia con la que están construidas las culturas (Vidal 2008: 83).

En ce qui concerne les études postcoloniales, il convient de mentionner les travaux d'Ovidi Carbonell de la Universidad de Salamanca. Le postcolonialisme en traductologie s'organise selon trois axes: la traduction en tant que moyen de colonisation; la réception des œuvres traduites dans une situation de relations de pouvoir inégales; et la traduction comme arme de déstabilisation du pouvoir colonialiste. Les ouvrages de Carbonell $(1997$; 1999) présentent une excellente analyse pour toute personne voulant faire ses premiers pas dans ce domaine.

\subsection{Traduction et étude de corpus traductologiques}

Dans l'espace interdisciplinaire où se développent les études de traduction, il n'est pas étonnant de retrouver les nouvelles technologies, les travaux empiriques et les recherches s'inspirant de la linguistique de corpus. C'est à Mona Baker, disciple de John Sinclair, et à ses élèves, Sara Laviosa et Dorothy Kenny, que l'on doit les travaux pionniers articulant linguistique de corpus et traduction. Dès 1995, Baker introduit les termes clés à prendre en compte dans cette approche (Baker 1995; 1996), qui seront précisés ultérieurement par Laviosa (1997; 1998) et Kenny (2001).

Les corpus se caractérisent par le fait qu'ils sont en format électronique, qu'ils peuvent intégrer des textes écrits et oraux et qu'ils peuvent prendre une ampleur extraordinaire. Les corpus informatisés sont utilisés dans la comparaison interlinguistique (études contrastives), ainsi que dans l'élaboration de dictionnaires et pour le développement de la traduction automatique. La CBMT - Corpus Based Machine Translation - qui se fonde sur l'usage de corpus textuels parallèles offre la possibilité d'alimenter les mémoires de traduction, les recherches en traduction automatique (MT) et les études statistiques.

Les programmes les plus complexes d'utilisation de corpus électroniques incluent des protocoles qui permettent de travailler avec deux types de corpus multilingues différents: parallèles et comparables. D’après Baker (1995), les corpus parallèles consolident le caractère descriptif de la traductologie actuelle et les travaux menés jusqu'à nos jours permettent de confirmer quatre universaux de la traduction: l'explicitation, la simplification, la normalisation et la convergence.

En Espagne, plusieurs chercheurs se sont penchés sur les travaux de corpus appliqués à la traduction, mais ceux-ci n'ont pas encore atteint leur plein développement. Toutefois, il convient de souligner les travaux de Gloria Corpas, de la Universidad de Málaga $(2000 ; 2003 ; 2007$; 2008) et de Mikel Forcada, de la Universidad de Alicante (2009), ainsi que ceux d'autres chercheurs tels que Álvarez (2001), Bravo et Fernández (1998) ou Calzada (2008)².

\subsection{Théories cognitives}

La nature de l'activité mentale du traducteur ou de l'interprète ainsi que la recherche du sens non verbal, qui dépend de la structuration cognitive liée à la connaissance du monde tout autant que de celle liée au contexte, ont intéressé de nombreux chercheurs. Parmi les premières écoles ayant traité de ce thème se trouve l'ÉSIT, avec la 
théorie du sens transmise en Espagne grâce aux travaux de Hurtado, Tricás ou Sáez. Calzada (2007: 205) cite Kussmaul et Kilary («Kussmaul analiza el proceso traductor; Kilary [...] pauta, sobre todo, el proceso de aprendizaje desde un punto de vista, para muchos, novedoso»), dont les travaux ont ouvert de nouveaux axes de recherche, notamment grâce à l'usage des protocoles de verbalisation (think aloud protocol; Kilary 1995).

L'importance de l'influence des facteurs cognitifs en traduction est une constante dans divers travaux publiés en Espagne, des années 1990 jusqu'à nos jours. Hurtado (2001), évoquant la théorisation de ce concept par l'ÉSIT (Bell, Kilary et Wills), affirme que la traduction est bien une opération cognitive, qui, en se situant sur le plan mental et dans le cadre plus large de la psychologie cognitive, s'intéresse aux difficultés de traduction ainsi qu'à leur résolution:

los enfoques cognitivos hoy por hoy no han producido mucho material específico del proceso de traducción y casi todo lo que estudiamos en el campo de la traducción originado en estos enfoques es aplicable en general a cualquier proceso de comunicación (Mayoral 1999: 102).

Soulignons encore les recherches menées par Ricardo Muñoz (Universidad de Granada), particulièrement prometteuses pour le développement de cette approche théorique (Muñoz 1993; 1994; 1995; 1996; 2007 ; 2008 et Fernández et Muñoz 2008), ainsi que ceux de Macizo (2003) et Padilla (Padilla, Macizo et al. 2007). Le groupe de recherche constitué par Muñoz, PETRA, vise à développer des recherches en ce sens:

Se trata de acometer la empresa de desarrollar una teoría traductológica basada en la investigación empírica, en un proyecto didáctico entre la reflexión filosófica y la observación de la realidad (Muñoz Martín 2008: 65).

Un des aspects importants de la recherche traductologique en matière de facteurs cognitifs est son caractère ouvert aux différentes conceptions de la traduction, comme le souligne Muñoz:

A diferencia de otras escuelas la aproximación cognitiva no rechaza las demás sino que sirve de marco a todas ellas, integrándolas como diversos puntos de análisis en una teoría general de la traducción. La teoría cognitiva se concibe como marco integrador de avances (Muñoz, texte inédit, cité par Mayoral 2001: 102).

\subsection{Autres approches: la traduction sous l'angle herméneutique et la traduction comme "technologie»}

Nous terminerons en présentant brièvement les réflexions de deux théoriciens, Emilio Ortega (Universidad de Málaga) et Roberto Mayoral (Universidad de Granada). Ortega, s'inspirant des contributions de Nida, de Schökel et de Ricœur, situe sa réflexion, dont il fait état dans Apuntes para una teoría hermenéutica de la traducción (1996), dans le cadre d'une théorie herméneutique de la traduction. Il retient de Nida et de Schökel les postulats sur la traduction de textes bibliques, et de Ricœur, les trois paramètres suivants: l'auteur, le monde évoqué par le texte et le lecteur. L'objectif de cette proposition est de créer une approche méthodologique fondée sur la théorie, mais qui soit viable dans la pratique: 
[...] que ésta consiste en un proceso de interpretación del texto original que conlleva, siguiendo a Nida y Schökel, tres etapas diferentes: análisis comprensión / interpretación y reestructuración / recreación. Por otro lado dichas etapas han de llevarse a cabo atendiendo a tres variables (Ricœur) que convergen en el texto: autor, lector y mundo del texto, y como consecuencia de todo ello el traductor habrá de optar, según la distinción de Schleiermacher, entre acercar el texto al lector o acercar el lector al texto una vez analizadas las interrelaciones existentes entre las tres variables que convergen en el texto objeto de traducción (Ortega 1996: 117-118).

Quant à Mayoral, Aspectos epistemológicos de la traducción (2001), qui associe la traduction à un processus technologique, il préconise une vision très large:

La definición del objeto de estudio de la traducción no es la definición de un proceso natural al que se presupone inalterabilidad sino la definición de un proceso tecnológico que se encuentra en continua evolución y cambio. [...] Necesitamos por tanto definiciones abiertas, que sea posible modificar tanto para contener las nuevas realidades (interpretación de lengua de signos, multimedia, producción de textos) como para desechar las que han dejado de mostrarse útiles y necesarias. Tanto para la traducción como para otras tecnologías, como el lenguaje o la literatura, nos mostramos partidarios de definiciones lo más inclusivas posibles que funcionen tanto por intensión (explicación) como por extensión (enumeración de los elementos que la componen), dejando esa enumeración abierta. Así una propuesta de definición de este tipo podría tomar una forma como la siguiente (nuestra formulación) (Mayoral 2001: 46).

C'est ainsi qu'il propose la définition suivante:

a. proceso comunicativo entre dos o más sistemas A y B (sistemas lingüísticos, culturales, semióticos, mediáticos, logográficos, etc.) diferentes en el que el mensaje $\mathrm{B}$ se deriva del mensaje A de alguna forma (mediante identidad, equivalencia, evocación, inspiración, alusión, referencia, comentario, resumen, paráfrasis, narración, fragmentación, adaptación, redacción, etc.), pudiendo el objetivo de la comunicación en ambos sistemas A y B ser diferente o idéntico; b. el resultado o producto del proceso anterior; c. el proceso mental seguido por la persona o el proceso seguido por la máquina que traducen (Mayoral 2001: 46).

Mayoral conclut en s'inscrivant en faux contre plusieurs des orientations mentionnées plus haut:

La práctica de la traducción es una tecnología, por lo que la disciplina que la estudie no puede ser una ciencia [...]. La traducción como proceso mental o cognitivo [...] resulta hoy por hoy inobservable [...]. Los Estudios de Traducción, aparte del esfuerzo gigantesco de constituirse en una disciplina aparte, han aportado realmente poco al conocimiento de la traducción [...] (Mayoral 2001: 131-133).

\section{Conclusion}

L'objectif du présent article était de rendre compte des travaux les plus importants des traductologues espagnols sans prendre parti pour aucun, mais plutôt afin de permettre au lecteur de se faire une opinion et d'approfondir, librement, les approches présentées. Tout comme nos recherches ont montré qu'il est vain de spéculer sur l'identité véritable de l'auteur - Avellaneda - du Don Quichotte apocryphe, il ne s'agissait pas pour nous de présenter tous les chercheurs espagnols, mais bien de montrer que la traductologie, en Espagne, fait l'objet d'une production importante, 
théorique et appliquée, qui constitue une contribution scientifique de valeur que nous pourrions résumer aux principaux aspects suivants:

- Le développement d'approches théoriques de la traduction en tant qu'ensemble de connaissances scientifiques systématisées;

- L'application pratique à l'espagnol de théories novatrices et parfois complémentaires au domaine didactique;

- L'adoption par les chercheurs espagnols de théories, hypothèses et études de traduction menées en Europe et en Amérique du Nord. Devenus de bons connaisseurs des dites théories et engagés dans une relation professionnelle avec leurs collègues, ces chercheurs remettent en question certains postulats, et développent, individuellement ou en groupe, voire créent de nouvelles avenues traductologiques;

- La pluralité d'approches et de définitions de la traduction: accords et divergences qui enrichissent les études de traduction.

En guise de conclusion, deux citations qui illustrent les pôles opposés du vaste débat que l'Espagne a entrepris en traductologie:

La formación de traductores inspirada en la existencia de principios teóricos superiores no puede llevar a ninguna parte (Mayoral 2001: 134)

Conviene que traductólogos y pedagogos profundicen de manera intensiva y extensiva, en los enfoques teóricos de la traducción. Cuando más sepamos sobre el fenómeno traductor, tanto mejor podremos enseñarlo. Cuantos más datos lingüísticos, comunicativos, funcionalistas, descriptivistas, éticos, cognitivos, emocionales, computacionales, etc., tengamos sobre el proceso y el producto traductor más información podremos trasladar a los futuros traductores. De manera que la teoría redundará en beneficio de la práctica y la práctica resulta imprescindible para enriquecer, contrastar y probar o refutar la teoría (Calzada 2007: 225-226).

\section{NOTES}

1. Santoyo, Julio César. Communication personnelle, courrier à l'auteur du 21 septembre 2009.

2. Calzada, María, dir. (2005-2008): Corpus comparables y paralelos de discursos de los Parlamentarios europeos. Proyecto financiado por el Ministerio de Educación Español.

\section{RÉFÉRENCES}

Álvarez, Alberto (2001): Estilística comparada da traducción: proposta metodológica e aplicación práctica ó estudio do corpus TEXTRA de traduccións do inglés ó galego. Vigo: Universidade de Vigo.

BAKer, Mona (1995): Corpora in Translation Studies: An Overview and Some Suggestions for Future Research. Target. 7(2):223-243.

BAKer, Mona (1996): Corpus-Based Translation Studies: The Challenges that Lie Ahead. In: Harold Somers, dir. Terminology, LSP and Translation Studies in Language Engineering in Honour of Juan C. Sager. Amsterdam: John Benjamins, 175-188.

Bravo, Jose María et Fernández, Purificación (1998): La lingüística de corpus, las nuevas tecnologías de la información y los Estudios de Traducción en la década de 1990. In: Purificación Fernández Nistal et José María Bravo gonzalo, dir. La traducción: orientaciones lingüísticas y culturales. Valladolid: Universidad de Valladolid, 205-257.

Calzada, María (2007): El espejo traductológico. Barcelona: Octaedro.

Carbonell, Ovidi (1997): Traducir al otro. Traducción, exotismo, poscolonianismo. Cuenca: Universidad de Castilla-la-Mancha.

Carbonell, Ovidi (1999): Traducción y cultura. De la ideología al texto. Salamanca: Colegio de España. 
Corpas, Gloria (2000): Traducir con corpus: de la teoría a la práctica. In: Joaquín García Palacios et Maria Teresa Fuentes Morón, dir. Texto, terminología y traducción. Salamanca: Ediciones Almar, 189-226.

Corpas, Gloria (2003): Diseño de un tipologizador para la traducción jurídica: del corpus al prototipo. In: Gloria CoRPAs, dir. Recursos documentales y tecnológicos para la traducción del discurso jurídico (español, alemán, inglés, italiano, árabe). Granada: Comares, 33-58.

Conpas, Gloria (2007): El concepto de representabilidad en lingüística de corpus: aproximación teórica y consecuencia para la traducción. Málaga: Universidad de Málaga.

CoRpas, Gloria (2008): Investigar en Corpus en traducción: los retos de un nuevo paradigma. Frankfurt: Peter Lang.

Elena, Pilar (1990): Aspectos teóricos y prácticos de la traducción alemán-español. Salamanca: Universidad de Salamanca.

Elena, Pilar (1994): Curso práctico de traducción general alemán-español. Salamanca: Universidad de Salamanca.

Elena, Pilar (2001): El traductor y el texto. Curso básico de traducción general (alemán-español). Madrid: Ariel.

Elena, Pilar et De Kock, Josse, dir. (2006): Gramática y traducción. Salamanca: Universidad de Salamanca.

Even-Zohar, Itamar (1999): Factores y dependencias en la cultura. Una revisión de las teorías de los polisistemas. In: Montserrat IgLesias, dir. Teorías de los polisistemas. Madrid: Arco / Libros, 23-52.

Fernández, María Manuela et Muñoz, Ricardo, dir. (2008): Aproximaciones cognitivas al estudio de la traducción e interpretación. Granada: Comares.

ForCADA, Mikel (2009): Apertium: traducció automàtica de codi obert per a les llengües romàniques. Linguamática. 1:13-23.

FUNDACIÓN AlFONSO X El SABio, dir. (1987): Problemas de la traducción. Mesa redonda 1983. Madrid: Fundación Alfonso X el Sabio.

García Yebra, Valentín (1982): Teoría y práctica de la traducción. Madrid: Gredos.

García Yebra, Valentín (1983/1989): En torno a la traducción. 2e éd. Madrid: Gredos.

García Yebra, Valentín (1994): La traducción: Historia y teoría. Madrid: Gredos.

García Yebra, Valentín (1999): Diccionario de galicismos prosódicos y morfológicos. Madrid: Gredos.

García Yebra, Valentín (2006): Experiencias de un traductor. Madrid: Gredos.

Hurtado, Amparo, dir. (1996) : La enseñanza de la traducción. Castellón: Universitat de Castellón.

Hurtado, Amparo, dir. (1999): Enseñar a traducir. Metodología en la formación de traductores e intérpretes. Barcelona: Gedisa.

Hurtado, Amparo (2001): Traducción y Traductología. Introducción a la Traductología. Madrid: Cátedra.

Kenny, Dorothy (2001): Lexis and Creativity. A Corpus Based Study. Manchester: St. Jerome.

Kilary, Donald (1995): Pathways to Translation. Pedagogy and Process. Kent: The Kent State University Press.

LaviosA, Sara (1997): How comparable can comparable corpora be? Target. 9(2):289-319.

Laviosa, Sara (1998): The English Comparable Corpus. A Resource and a Methodology. In: Lyne Bowker, Michael Cronin, Dorothy Kenny, et al., dir. Unity in Diversity: Current Trends in Translation Studies. Manchester: St. Jerome, 101-112.

Lorenzo, Emilio (1987): Prefacio. In: Fundación alfonso x EL SABio, dir. Problemas de la traducción. Mesa redonda 1983. Madrid: Fundación Alfonso X el Sabio, 5-6.

Lvóvskaya, Zinaida (1997): Problemas actuales de la traducción. Granada: Método Ediciones.

Macizo, Pedro (2003): Procesos cognitivos en la traducción: comprensión y memoria de trabajo. Thèse de doctorat non publiée. Granada: Universidad de Granada. 
MAYORAL, Roberto (1999) : La traducción de la variación lingüistica. Valladolid: Universidad de Valladolid.

Mayoral, Roberto (2001): Aspectos epistemológicos de la traducción. Castellón: Universidad de Castellón.

Merino, Raquel (1994): Traducción, tradición y manipulación: Teatro inglés en España 19501990. León / Bilbao: Universidades de León y del País Vasco.

Merino, Raquel (2001): Presentación de la base de datos TRACE (Traducciones censuradas inglés-español). In: Eterio Pajares, Raquel Merino et José Miguel Santamaria, dir. Transvases culturales. Literatura, cine y traducción. Vol. 3. Vitoria: Universidad del País Vasco, 287-295.

Moya, Virgilio (2004): La selva de la traducción. Madrid: Cátedra.

Muñoz Martín, Ricardo (1993): A Cognitive Theory of Professional Translation. Thèse de doctorat. Berkeley: University of California.

MuÑoz MarTín, Ricardo (1994): El significado de las teorías lingüísticas de la traducción: hacia una aproximación cognitiva. Sendebar. 5:67-83.

Muñoz Martín, Ricardo (1995): Lingüística para traducir. Barcelona: Teide.

Muñoz Martín, Ricardo (1996): Lingüística per tutti y salga el Sol por Antequera. Trans. 1:35-142.

MuÑoz Martín, Ricardo (2007): Traducción cognitiva y traducción empírica. In: Gerd WotJAK, dir. Quo vadis, Translatologie? Ein halbes Jahrhundert universitäre Ausbildung von Dolmetschern und Übersetzern in Leipzig. Berlin: Franck \& Timme, 267-278.

Muñoz Martín, Ricardo (2008) : Apuntes para una traductología cognitiva. In: Luis Pegenaute, Janet de CASARIs, Mercè TricÁs et Elisenda Bernal, dir. La traducción del futuro: mediación lingüistica y cultural en el siglo XXI. Barcelona: PPU-AIETTI, 65-74.

Navarro, Fernando (1996): Manual de bibliografía española de traducción e interpretación. Diez años de historia 1985-1995. Alicante: Universidad de Alicante.

Ortega, Emilio (1996): Apuntes para una teoría hermenéutica de la traducción. Málaga: Universidad de Málaga.

Ortega, Emilio, dir. (2004): Panorama actual de la investigación en traducción e interpretación. Granada: Atrio.

Padilla, Presentación, Macizo, Pedro et Bajo, Maria José (2007): Tareas de traducción e interpretación desde la perspectiva cognitiva. Una propuesta integradora. Granada: Atrio.

RABADÁN, Rosa (1991): Equivalencia y traducción. Léon: Universidad de León.

RABADÁN, Rosa, dir. (2000): Traducción y censura Inglés-Español 1939-1985. Estudio preliminar. Léon: Universidad de León.

Rabadán, Rosa et Merino, Raquel (2004): Introducción a la edición española. In: Gideon Toury, dir. Los estudios descriptivos de traducción y más allá - Metodología de la investigación en los Estudios de Traducción. Madrid: Cátedra, 17-40.

SÁnchez Trigo, Elena (2002): Teoría de la traducción: convergencias y divergencias. Vigo: Universidade de Vigo.

SANToYo, Julio César (1983) : La cultura traducida. Lección inaugural del curso académico 19831984. León: Universidad de León.

Santoyo, Julio César (1983): A propósito del término «translema». In: Asociación Española de Lingüística Aplicada (AESLA). Actas del I Congreso Nacional de Lingüística Aplicada. Tendencias actuales en las aplicaciones de la Lingüística. Madrid: SGEL, 255-266.

Santoyo, Julio César (1985/1996) : El delito de traducir. $3^{e}$ éd. Léon: Universidad de León.

SAntoyo, Julio César (1987): Teoría y crítica de la traducción: Antología. Barcelona: Universitat Autònoma de Barcelona.

SAntoyo, Julio César (1996): Bibliografía de la traducción en español, catalán, gallego y vasco. Léon: Universidad de León.

Santoyo, Julio César (1997): Del scriptorium a la Academia: Acerca de cómo, dónde, cuándo y por qué la reflexión teórica se ha ido incorporando al quehacer traductor. Parallèles. 19:99-112. 
Toury, Gideon (1995): Descriptive Translation Studies and Beyond. Amsterdam: John Benjamins.

Toury, Gideon (1997): What Lies beyond Descriptive Translation Studies? In: Miguel Ángel Vega, dir. La palabra vertida. Madrid: Universidad Complutense de Madrid, 69-80.

Toury, Gideon (2004): Los estudios descriptivos de traducción y más allá - Metodología de la investigación en los Estudios de Traducción. (Traduit par Rosa Rabadán et Raquel Merino). Madrid: Cátedra.

TricÁs, Mercedes (1987a) : Lingüística textual y traducción. In: FundaCión ALFONSO x EL SABIO, dir. Problemas de la traducción. Mesa redonda 1983. Madrid: Fundación Alfonso X el Sabio, 131-154.

TRICÁs, Mercedes (1987b): La traducción de los conectores del discurso (con referencia a la traducción del francés al castellano). Parallèles. 8:27-35.

Tricás, Mercedes (1990): L’argumentation concessive française et espagnole: Le transfert en espagnol de quelques connecteurs (pourtant, quand même, même si, or ...) et d'autres formules concessives. Meta. 35(3):529-537.

Tricás, Mercedes (1991): Polifonía discursiva y traducción: propuestas de tratamiento de los enunciadores que recuperan otro universo sociolingüístico. In: María Luisa DonaIre et Francisco LAfarga, dir. Traducción y adaptación cultural España-Francia. Oviedo: Universidad de Oviedo, 513-528.

Tricás, Mercedes (1995): La traducción al español de los valores interactivos y argumentativos del conector «alors». Iberica. 5:207-224.

TricÁs, Mercedes (1995/2003): Manual de traducción Francés-Castellano. $2^{e}$ éd. Barcelona: Gedisa.

TRICÁs, Mercedes (2008) : Construir (equivalencias): del texto a la representación intercultural. In: Luis Pegenaute, Janet de casaris, Mercè Tricás et Elisenda Bernal, dir. La traducción del futuro: mediación lingüística y cultural en el siglo XXI. Barcelona: PPU-AIETTI, $89-100$.

Vega Cernuda, Miguel Ángel (1994/2004): Textos clásicos de teoría de la traducción. $2^{e}$ éd. Madrid: Cátedra.

VIDAL, África (1995): Traducción, manipulación, desconstrucción. Salamanca: Editorial Colegio de España.

VIDAL, África (1998): El futuro de la traducción. Valencia: Institut Alfons El Magnànim.

VIDAL, África (2005): En los límites de la traducción. Granada: Comares.

VIDAL, África (2008): Traducir en el siglo XXI : nuevos retos de la investigación traductológica. In: Javier Gómez Montero, dir. Nuevas pautas de traducción literaria. Madrid: Visor Libros, 75-86. 